Peter Jørgensen

\title{
Existence of Gorenstein projective resolutions and Tate cohomology
}

Received May 19, 2005 and in revised form September 16, 2005

\begin{abstract}
Existence of proper Gorenstein projective resolutions and Tate cohomology is proved over rings with a dualizing complex. The proofs are based on Bousfield Localization which is originally a method from algebraic topology.
\end{abstract}

Keywords. Dualizing complex, Gorenstein homological algebra, Gorenstein projective precover

\section{Introduction}

This paper proves two ring-theoretical results: existence of proper Gorenstein projective resolutions and existence of Tate cohomology over fairly general rings.

The proofs use Bousfield Localization which, like many modern conveniences, was invented by algebraic topologists (see [4]). It has so far not been used extensively in ring theory, but the results of this paper show that, when phrased as an existence theorem for adjoint functors, it is a very natural ring theory tool.

The proof of existence of proper Gorenstein projective resolutions solves an open problem in Gorenstein homological algebra.

Gorenstein projective resolutions. Classical homological algebra might be viewed as being based on projective modules. In relative homological algebra, one replaces the projective modules with some other class of modules (see [6]). One choice is to take the Gorenstein projective modules; this results in Gorenstein homological algebra which goes back to [1]. These modules have the form $G=\operatorname{Ker}\left(E^{1} \stackrel{\partial_{E}^{1}}{\rightarrow} E^{2}\right)$ where $E$ is a complete projective resolution, that is, an exact complex of projective modules which stays exact when one applies the functor $\operatorname{Hom}(-, Q)$ for any projective module $Q$.

An augmented proper Gorenstein projective resolution of a module $M$ is an exact sequence

$$
\cdots \rightarrow G_{2} \rightarrow G_{1} \rightarrow G_{0} \rightarrow M \rightarrow 0
$$

P. Jørgensen: Department of Pure Mathematics, University of Leeds, Leeds LS2 9JT, United Kingdom; e-mail: popjoerg@ @aths.leeds.ac.uk; URL: http://www.maths.leeds.ac.uk/popjoerg

Current address: School of Mathematics and Statistics, Newcastle University, Newcastle upon Tyne NE1 7RU, United Kingdom; e-mail: peter.jorgensen@ncl.ac.uk;

URL: http://www.staff.ncl.ac.uk/peter.jorgensen/

Mathematics Subject Classification (2000): 13D02, 16E05, 18G25, 20J06 
where the $G_{i}$ are Gorenstein projective modules, which stays exact when one applies the functor $\operatorname{Hom}(\widetilde{G},-)$ for any Gorenstein projective module $\widetilde{G}$. The complex

$$
G=\cdots \rightarrow G_{2} \rightarrow G_{1} \rightarrow G_{0} \rightarrow 0 \rightarrow \cdots
$$

is then called a proper Gorenstein projective resolution of $M$.

Gorenstein homological algebra has been developed to an advanced level (see [5]). One of the central points is the ability of the theory to recognize Gorenstein rings. A noetherian local commutative ring $A$ is called Gorenstein if it is Cohen-Macaulay and has an irreducible parameter ideal (see [22, Thm. 18.1]), and it turns out that $A$ is Gorenstein if and only if each $A$-module has a proper Gorenstein projective resolution with $G_{i}=0$ for $i \gg 0$.

One problem of the theory is that existence of proper Gorenstein projective resolutions is not obvious in general, because of the condition that (1) must stay exact when one applies the functor $\operatorname{Hom}(\widetilde{G},-)$. We generally know little about the structure of the class of Gorenstein projective modules, so the precise content of this condition is unclear.

A possible solution is to drop the condition, and this approach has been taken by a number of authors (see [5]). The resulting resolutions are then simply called Gorenstein projective resolutions, and they always exist. For instance, any projective resolution can be used since the projective modules are also Gorenstein projective.

Unfortunately, dropping the condition that (1) must stay exact when one applies the functor $\operatorname{Hom}(\widetilde{G},-)$ for any Gorenstein projective module $\widetilde{G}$ ignores an important point: The purpose of this condition is that it makes the resolution (2) unique up to chain homotopy (see [11, Prop. 2.2]). This in turn means that (2) can be used to define the Gorenstein version of derived functors. Without the condition that (1) stays exact under the functor $\operatorname{Hom}(\widetilde{G},-)$, any such definition fails, and the theory must lead a shadow existence without derived functors.

However, the present paper solves the problem by proving the existence of proper Gorenstein projective resolutions over fairly general rings. This is done by showing that the resolutions exist under one simple assumption-the existence of a certain adjoint functor $e^{!}$- and by using Bousfield Localization to show that $e^{!}$exists if the ground ring has a dualizing complex. This covers many rings arising in practice. For instance, any local ring of a scheme of locally finite type over a field has a dualizing complex. Other types of rings are also covered; see Remark 1.1 .

In fact, it may even be that some form of Bousfield Localization can be used to show that the functor $e^{!}$exists over any ring and hence that proper Gorenstein projective resolutions exist in general, but this is not clear to me at the moment.

Since this paper makes proper Gorenstein projective resolutions available over most rings which occur in practice, I propose to simplify the terminology by dropping the word "proper". So for the rest of the paper, an augmented Gorenstein projective resolution of $M$ will be an exact sequence (1) where the $G_{i}$ are Gorenstein projective, which stays exact when one applies the functor $\operatorname{Hom}(\widetilde{G},-)$ for any Gorenstein projective module $\widetilde{G}$. When this is given, (2) will be called a Gorenstein projective resolution of $M$. 
Tate cohomology. For the second result of the paper recall that, originally, Tate cohomology was defined for representations of finite groups. Generalizing this, I will show, again under the assumption that the adjoint functor $e^{!}$exists, that it is possible to define Tate cohomology groups

$$
\widehat{\operatorname{Ext}}^{i}(M, N)
$$

for any modules $M$ and $N$, so that classical Tate cohomology is the special case $\widehat{\operatorname{Ext}}_{k G}^{i}(k, N)$. Moreover, it will be established that the Tate cohomology groups have reasonable basic properties, and it will be shown that there is a close connection between Tate cohomology and Gorenstein homological algebra in the form of a long exact sequence

$$
0 \rightarrow \operatorname{Ext}_{\mathscr{G}}^{1}(M, N) \rightarrow \operatorname{Ext}^{1}(M, N) \rightarrow \widehat{\operatorname{Ext}}^{1}(M, N) \rightarrow \cdots
$$

where the $\operatorname{Ext}_{\mathscr{G}}^{i}$ are Gorenstein Ext groups defined by

$$
\operatorname{Ext}_{\mathscr{G}}^{i}(M, N)=\mathrm{H}^{i} \operatorname{Hom}(G, N),
$$

where $G$ is a Gorenstein projective resolution of $M$. The $\operatorname{Ext}_{\mathscr{G}}^{i}$ are precisely the Gorenstein derived functors of Hom.

Relations to the literature. The notion of Gorenstein projective modules is complemented by notions of Gorenstein flat and Gorenstein injective modules, and the existence of Gorenstein flat and Gorenstein injective resolutions was established in [7] and [8]. Some other injective cousins of the results of this paper are given in [18].

On the other hand, it was proved in [25] that if one restricts to finitely generated modules, then Gorenstein projective resolutions do not exist in general.

Precursors to the present results can be found in [3] and [26]. However, these papers only work with modules of finite Gorenstein projective dimension, and this restricts the real scope of the theory to Gorenstein rings whereas the present paper works for much more general rings.

A generalized version of Tate cohomology already exists in the form of Tate-Vogel cohomology (see [9] and [21]). However, this theory differs from the Tate cohomology which will be developed here (see [12, Rmk. 6.5]). I refer to the present theory simply as "Tate cohomology" because I think of it as the most direct generalization of the classical theory, since, like the classical theory, it is based on complete projective resolutions (cf. Definition 3.2 and Remark 3.3.

Perspectives. As pointed out, this paper's result on Gorenstein projective resolutions permits the definition of Gorenstein derived functors. This was not previously possible, and should prove useful in Gorenstein homological algebra.

Applications of the present Tate cohomology theory are as yet more speculative. As mentioned, the present theory generalizes the Tate cohomology of [3], in which alternative Betti and Bass numbers based on Tate cohomology are considered and proved to exhibit surprising patterns which are impossible in classical Betti and Bass numbers. It would be obvious to try something similar for more general rings, using the present theory.

Also, [20] and [21] consider invariants related to Betti and Bass numbers which are based on Tate-Vogel cohomology, and use them to introduce other new invariants and to 
prove properties of syzygy modules. Again, something similar may be possible using the present Tate cohomology theory.

The paper is organized as follows. Section 1 uses Bousfield Localization to show the existence of the adjoint functor $e^{!}$over rings with a dualizing complex. Section 2 shows the existence of Gorenstein projective resolutions when $e^{!}$exists. And Section 3 defines Tate cohomology groups, shows some basic properties, and shows that the Tate cohomology groups fit into the exact sequence (3).

\section{Bousfield Localization}

This section uses Bousfield Localization to show the existence of a certain adjoint functor $e^{!}$over rings with a dualizing complex.

Remark 1.1. Dualizing complexes are popular gadgets in homological algebra. I shall give the precise definition in Setup 1.4 for noetherian commutative rings and in Setup 1.4 for right-noetherian algebras over a field. But I would like already here to point out that many rings have dualizing complexes.

For instance, a noetherian local commutative ring has a dualizing complex if and only if it is a quotient of a Gorenstein noetherian local commutative ring, by [16, Thm. 1.2]. It follows that, as mentioned in the introduction, any local ring of a scheme of locally finite type over a field has a dualizing complex. By the Cohen structure theorem, it also follows that any complete noetherian local commutative ring does.

Some important types of non-commutative noetherian algebras are also known to have dualizing complexes. For example, complete semi-local PI algebras do by [27, Cor. 0.2], and filtered algebras do by [28, Cor. 6.9] if their associated graded algebras are noetherian and connected, and either PI, FBN, or with enough normal elements.

Definition 1.2. If $A$ is a ring, then $\mathrm{E}(A)$ denotes the class of complete projective resolutions of A-left-modules. So a complex of A-left-modules $E$ is in $\mathrm{E}(A)$ if it consists of projective A-left-modules, is exact, and has $\operatorname{Hom}_{A}(E, Q)$ exact for each projective A-left-module $Q$.

Remark 1.3. I will view $\mathrm{E}(A)$ as a full subcategory of $\mathrm{K}$ (Pro $A)$, the homotopy category of complexes of projective $A$-left-modules. The inclusion functor will be denoted

$$
e_{*}: \mathrm{E}(A) \rightarrow \mathrm{K}(\operatorname{Pro} A) .
$$

Setup 1.4. Let $A$ be a noetherian commutative ring with a dualizing complex $D$. That is,

(i) The cohomology of $D$ is bounded and finitely generated over $A$.

(ii) The injective dimension $\operatorname{id}_{A} D$ is finite.

(iii) The canonical morphism $A \rightarrow \operatorname{RHom}_{A}(D, D)$ in the derived category $\mathrm{D}(A)$ is an isomorphism.

Setup 1.5. Let $D \stackrel{\simeq}{\rightarrow} I$ be an injective resolution for which $I$ is a bounded complex.

See [10, Chp. V] for background on dualizing complexes. 
Remark 1.6. Since $A$ has a dualizing complex, it has finite Krull dimension by [10, Cor. V.5.2], so by [24, Seconde partie, cor. (3.2.7)], each flat $A$-module has finite projective dimension.

The following lemma uses $I$, the injective resolution of the dualizing complex $D$.

Lemma 1.7. Let $P$ be a complex of projective A-modules. Then

$\operatorname{Hom}_{A}(P, Q)$ is exact for each projective A-module $Q \Leftrightarrow I \otimes_{A} P$ is exact.

Proof. $\Rightarrow$ Suppose that $\operatorname{Hom}(P, Q)$ is exact for each projective module $Q$. To see that $I \otimes P$ is an exact complex, it is enough to see that

$$
\operatorname{Hom}(I \otimes P, J) \cong \operatorname{Hom}(P, \operatorname{Hom}(I, J))
$$

is exact for each injective module $J$.

It follows from [19, Thm. 1.2] that $\operatorname{Hom}(I, J)$ is a bounded complex of flat modules. Hence, $\operatorname{Hom}(I, J)$ is finitely built from flat modules in the homotopy category of complexes of $A$-modules, $\mathrm{K}(A)$, and so it is enough to see that $\operatorname{Hom}(P, F)$ is exact for each flat module $F$.

Since $F$ has finite projective dimension by Remark 1.6 , there is a projective resolution $\widetilde{P} \stackrel{\simeq}{\rightarrow} F$ with $\widetilde{P}$ bounded. Since $P$ consists of projective modules and both $\widetilde{P}$ and $F$ are bounded, this induces a quasi-isomorphism

$$
\operatorname{Hom}(P, \widetilde{P}) \simeq \operatorname{Hom}(P, F) .
$$

So it is enough to see that $\operatorname{Hom}(P, \widetilde{P})$ is exact.

But $\widetilde{P}$ is a bounded complex of projective modules, so it is finitely built from projective modules, so it is enough to see that $\operatorname{Hom}(P, Q)$ is exact for each projective module $Q$. And this holds by assumption.

$\Leftarrow$ Suppose that $I \otimes P$ is an exact complex. I must show that $\operatorname{Hom}(P, Q)$ is exact for each projective module $Q$.

First observe that by [2, Thm. (3.2)], there is an isomorphism

$$
Q \stackrel{\cong}{\rightrightarrows} \operatorname{RHom}(D, D \stackrel{\mathrm{L}}{\otimes} Q) \text {. }
$$

Of course, I can replace $D$ by $I$ to get

$$
Q \stackrel{\cong}{\rightarrow} \operatorname{RHom}(I, I \stackrel{\mathrm{L}}{\otimes} Q) .
$$

Here

$$
I \stackrel{\mathrm{L}}{\otimes} Q \cong I \otimes Q
$$

because $Q$ is projective. Moreover, $I \otimes Q$ is a bounded complex of injective modules so

$$
\operatorname{RHom}(I, I \stackrel{\mathrm{L}}{\otimes} Q) \cong \operatorname{RHom}(I, I \otimes Q) \cong \operatorname{Hom}(I, I \otimes Q) .
$$

So the isomorphism (4) in the derived category is represented by the chain map

$$
Q \rightarrow \operatorname{Hom}(I, I \otimes Q)
$$

which must accordingly be a quasi-isomorphism. 
Completing to a distinguished triangle in $\mathrm{K}(A)$ gives

$$
Q \rightarrow \operatorname{Hom}(I, I \otimes Q) \rightarrow C \rightarrow
$$

where $C$ is exact. Here $I$ and $I \otimes Q$ are bounded, so $\operatorname{Hom}(I, I \otimes Q)$ is bounded. As the same is true for $Q$, the mapping cone $C$ is also bounded.

Now, the distinguished triangle gives another distinguished triangle

$$
\operatorname{Hom}(P, Q) \rightarrow \operatorname{Hom}(P, \operatorname{Hom}(I, I \otimes Q)) \rightarrow \operatorname{Hom}(P, C) \rightarrow
$$

Here $\operatorname{Hom}(P, C)$ is exact because $P$ is a complex of projective modules while $C$ is a bounded exact complex. So to see that $\operatorname{Hom}(P, Q)$ is exact as desired, it is enough to see that $\operatorname{Hom}(P, \operatorname{Hom}(I, I \otimes Q))$ is exact.

However,

$$
\operatorname{Hom}(P, \operatorname{Hom}(I, I \otimes Q)) \cong \operatorname{Hom}(I \otimes P, I \otimes Q) .
$$

And this is exact because $I \otimes P$ is exact by assumption while $I \otimes Q$ is a bounded complex of injective modules.

Lemma 1.8. The homotopy category of complexes of projective A-modules, $\mathrm{K}(\operatorname{Pro} A)$, is a compactly generated triangulated category.

Proof. It is clear that $\mathrm{K}$ (Pro $A$ ) is triangulated. The ring $A$ is noetherian and hence coherent, and by Remark 1.6 each flat $A$-module has finite projective dimension. So K(Pro $A$ ) is compactly generated by [15, Thm. 2.4].

Combining Lemmas 1.7 and 1.8 with Bousfield Localization now gives existence of the adjoint functor $e^{!}$.

Proposition 1.9. The inclusion functor $e_{*}: \mathrm{E}(A) \rightarrow \mathrm{K}($ Pro $A)$ has a right-adjoint $e^{!}$: $\mathrm{K}(\operatorname{Pro} A) \rightarrow \mathrm{E}(A)$.

Proof. Consider the functor

$$
k(-)=\mathrm{H}^{0}\left((A \oplus I) \otimes_{A}-\right)
$$

from $\mathrm{K}(\operatorname{Pro} A)$ to $\mathrm{Ab}$, the category of abelian groups. This is clearly a homological functor respecting set indexed coproducts. Moreover,

$$
k\left(\Sigma^{i} P\right) \cong \mathrm{H}^{i}(P) \oplus \mathrm{H}^{i}\left(I \otimes_{A} P\right),
$$

where $\Sigma^{i}$ denotes the $i$ th suspension, so for $P$ to satisfy $k\left(\Sigma^{i} P\right)=0$ for each $i$ means

$$
\mathrm{H}^{i}(P)=0 \quad \text { and } \quad \mathrm{H}^{i}\left(I \otimes_{A} P\right)=0
$$

for each $i$. Using Lemma 1.7, this shows

$$
\left\{P \in \mathrm{K}(\operatorname{Pro} A) \mid k\left(\Sigma^{i} P\right)=0 \text { for each } i\right\}=\mathrm{E}(A) .
$$

That is, $\mathrm{E}(A)$ is the kernel of the homological functor $k$. 
One consequence of this is that $\mathrm{E}(A)$ is closed under set indexed coproducts. Hence [17, Lem. 3.5] says that for $e_{*}$ to have a right-adjoint is the same as for the Verdier quotient $\mathrm{K}(\operatorname{Pro} A) / \mathrm{E}(A)$ to have the property that each Hom set is in fact a set (as opposed to a class).

Now, the category $\mathrm{K}(\operatorname{Pro} A)$ is compactly generated by Lemma 1.8 . By [23, Lem. 4.5.13] with $\beta=\aleph_{0}$, this even implies that there is only a set of isomorphism classes of compact objects in $\mathrm{K}$ (Pro $A)$. Hence the version of Bousfield Localization given in [14, Thm. 4.1] applies to the functor $k$ on $\mathrm{K}(\operatorname{Pro} A)$, and shows that in $\mathrm{K}$ (Pro $A$ ) modulo the kernel of $k$, each Hom is a set. That is, in $\mathrm{K}(\operatorname{Pro} A) / \mathrm{E}(A)$ each Hom is a set, as desired.

The methods given above also apply to non-commutative algebras. Let the following setups replace Setups 1.4 and 1.5

Setup 1.4. Let $A$ be a left-coherent and right-noetherian $k$-algebra over the field $k$ for which there exists a left-noetherian $k$-algebra $B$ and a dualizing complex ${ }_{B} D_{A}$. That is, $D$ is a complex of $B$-left- $A$-right-modules, and

(i) The cohomology of $D$ is bounded and finitely generated both over $B$ and over $A^{\text {op }}$.

(ii) The injective dimensions $\operatorname{id}_{B} D$ and $\operatorname{id}_{A}$ op $D$ are finite.

(iii) The canonical morphisms

$$
A \rightarrow \operatorname{RHom}_{B}(D, D) \quad \text { and } \quad B \rightarrow \operatorname{RHom}_{A^{\mathrm{op}}}(D, D)
$$

in the derived categories $\mathrm{D}\left(A \otimes_{k} A^{\mathrm{op}}\right)$ and $\mathrm{D}\left(B \otimes_{k} B^{\mathrm{op}}\right)$ are isomorphisms.

Setup 1.5. Let $D \stackrel{\simeq}{\rightarrow} I$ be an injective resolution of $D$ over $B \otimes_{k} A^{\text {op }}$. Replace $I$ by a bounded truncation. This may ruin the property that $I$ is an injective resolution over $B \otimes_{k} A^{\text {op }}$, but because $\operatorname{id}_{B} D$ and $\operatorname{id}_{A}$ op $D$ are finite, I can still suppose that $I$ consists of modules which are injective both over $B$ and over $A^{\text {op }}$.

The definition of dualizing complexes over non-commutative algebras is taken from [28, Def. 1.1].

With Setups 1.4 and 1.5 replaced by Setups 1.4 and 1.5 , let me inspect the rest of this section. As the ground ring $A$ is now non-commutative, I must replace "module" by "left-module" throughout. Remark 1.6 also needs to be replaced by the following.

Remark 1.6. Under Setup 1.4, each flat $A$-left-module has finite projective dimension by [13].

After this, the proof of Lemma 1.7 goes through if one keeps track of left and right structures throughout, and the proofs of Lemma 1.8 and Proposition 1.9 also still work.

Hence I can sum up the results of this section in the following theorem.

Theorem 1.10. Consider either of the following two situations.

(i) A is a noetherian commutative ring with a dualizing complex (see Setup 1.4).

(ii) $A$ is a left-coherent and right-noetherian $k$-algebra over the field $k$ for which there exists a left-noetherian $k$-algebra $B$ and a dualizing complex ${ }_{B} D_{A}$ (see Setup 1.4 ). 
Then the inclusion functor

$$
e_{*}: \mathrm{E}(A) \rightarrow \mathrm{K}(\operatorname{Pro} A)
$$

has a right-adjoint

$$
e^{!}: \mathrm{K}(\operatorname{Pro} A) \rightarrow \mathrm{E}(A)
$$

\section{Gorenstein projective resolutions}

This section shows the existence of Gorenstein projective resolutions when the adjoint functor $e^{!}$exists.

Setup 2.1. For the rest of this paper, $A$ is a ring for which the inclusion functor $e_{*}$ : $\mathrm{E}(A) \rightarrow \mathrm{K}(\operatorname{Pro} A)$ has a right-adjoint $e^{!}: \mathrm{K}(\operatorname{Pro} A) \rightarrow \mathrm{E}(A)$.

Remark 2.2. The existence of the right-adjoint $e^{!}$is precisely the hypothesis under which the constructions of this paper work.

The functor $e^{!}$exists over fairly general rings; see Theorem 1.10 and Remark 1.1. As mentioned in the introduction, it may even be the case that $e^{!}$exists over any ring, but I do not know how to prove that.

Remark 2.3. If $P$ is a complex of projective $A$-left-modules, then $e^{!} P$ can be thought of as the best approximation to $P$ by a complete projective resolution.

Elaborating on this, if $M$ is an $A$-left-module with projective resolution $P$, then $e^{!} P$ can be thought of as the best approximation to $M$ by a complete projective resolution. This point will be made more precise in Lemma 3.6

Construction 2.4. If $P$ is a complex of $A$-left-modules, then for each $i$ there is a chain map

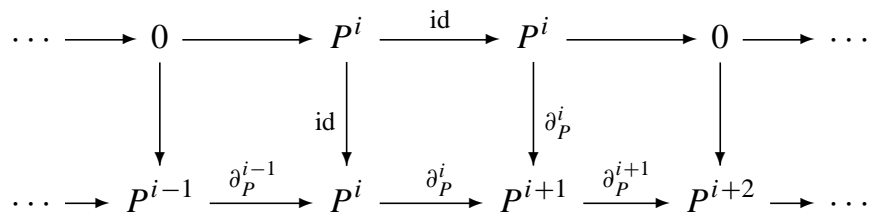

where the upper complex is null homotopic.

If $T \stackrel{t}{\rightarrow} P$ is now a chain map, then I can add the upper complex to $T$ and thereby change $t$ so that the $i$ th component $T^{i} \stackrel{t^{i}}{\rightarrow} P^{i}$ becomes surjective. Doing so does not change the isomorphism class of $t$ in $\mathrm{K}(A)$, the homotopy category of complexes of $A$ left-modules.

Construction 2.5. If $M$ is an $A$-left-module, then let $P$ be a projective resolution concentrated in non-positive cohomological degrees and consider the counit morphism $e_{*} e^{!} P \stackrel{\epsilon P}{\rightarrow} P$ in $\mathrm{K}(\operatorname{Pro} A)$. By applying Construction 2.4 in each degree, I can assume that $\epsilon_{P}$ is represented by a surjective chain map, so for

$$
F=e_{*} e^{!} P
$$


there is a short exact sequence of complexes

$$
0 \rightarrow K \rightarrow F \rightarrow P \rightarrow 0
$$

Note that since both $F$ and $P$ consist of projective modules, the sequence is semi-split (that is, split in each degree) and $K$ also consists of projective modules.

Lemma 2.6. Consider the complex $K$ from Construction 2.5. Then

$$
\operatorname{Hom}_{\mathrm{K}(\operatorname{Pro} A)}(E, K)=0
$$

for $E$ in $\mathrm{E}(A)$.

Proof. The chain map $F \rightarrow P$ represents the counit morphism

$$
e_{*} e^{!} P \stackrel{\epsilon_{P}}{\rightarrow} P
$$

which leads to a commutative diagram

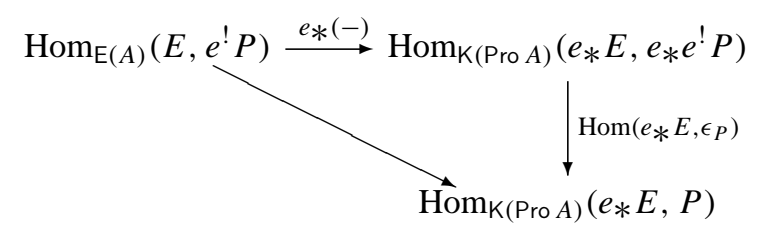

where the diagonal map is the adjunction isomorphism while the horizontal map is an isomorphism because $e_{*}$ is the inclusion functor of a full subcategory. The vertical map must therefore also be an isomorphism. That is,

$$
\operatorname{Hom}_{\mathrm{K}(\operatorname{Pro} A)}(E, F) \rightarrow \operatorname{Hom}_{\mathrm{K}(\operatorname{Pro} A)}(E, P)
$$

is an isomorphism.

Now, the short exact sequence from Construction 2.5 is semi-split and therefore gives a distinguished triangle

$$
K \rightarrow F \rightarrow P \rightarrow
$$

in $\mathrm{K}(\operatorname{Pro} A)$. Hence there is a long exact sequence consisting of pieces

$$
\operatorname{Hom}_{\mathrm{K}(\operatorname{Pro} A)}\left(\Sigma^{i} E, K\right) \rightarrow \operatorname{Hom}_{\mathrm{K}(\operatorname{Pro} A)}\left(\Sigma^{i} E, F\right) \rightarrow \operatorname{Hom}_{\mathrm{K}(\operatorname{Pro} A)}\left(\Sigma^{i} E, P\right) .
$$

Since $\Sigma^{i} E$ is in $\mathrm{E}(A)$ for each $i$, the second homomorphism here is of the type from equation (5), so is an isomorphism for each $i$. This implies $\operatorname{Hom}_{\mathrm{K}(\operatorname{Pro} A)}(E, K)=0$ as desired.

Remark 2.7. For the following lemma, recall that a Gorenstein projective A-left-module is a module of the form $G=\operatorname{Ker}\left(E^{1} \stackrel{\partial_{E}^{1}}{\rightarrow} E^{2}\right.$ ) where $E$ is in $\mathrm{E}(A)$ (cf. Definition 1.2).

It is not hard to see that each projective $A$-left-module is Gorenstein projective, but in general, there are other Gorenstein projective modules than these. For instance, over a noetherian local commutative Gorenstein ring, each maximal Cohen-Macaulay module is Gorenstein projective. 
Lemma 2.8. Consider the complex $K$ from Construction 2.5. Suppose that the sequence

$$
\cdots \rightarrow K^{i-2} \rightarrow K^{i-1} \stackrel{k}{\rightarrow} N \rightarrow 0
$$

obtained from $K$ is exact. Let $\widetilde{G}$ be Gorenstein projective and let $\widetilde{G} \stackrel{\widetilde{g}}{\rightarrow} N$ be a homomorphism. Then $\widetilde{g}$ lifts through $k$,

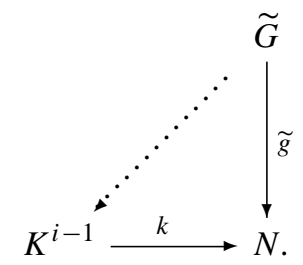

Proof. By (de)suspending, I can clearly pick a complex $E$ in $\mathrm{E}(A)$ with $\widetilde{G}=\operatorname{Ker}\left(E^{i} \stackrel{\partial_{E}^{i}}{\rightarrow}\right.$ $\left.E^{i+1}\right)$. Since $E$ is in $\mathrm{E}(A)$, the kernel of each differential in $E$ is Gorenstein projective, and it is not hard to see that there is a chain map $E \stackrel{e}{\rightarrow} K$ which fits together with $\widetilde{G} \stackrel{\widetilde{g}}{\rightarrow} N$ in a commutative diagram

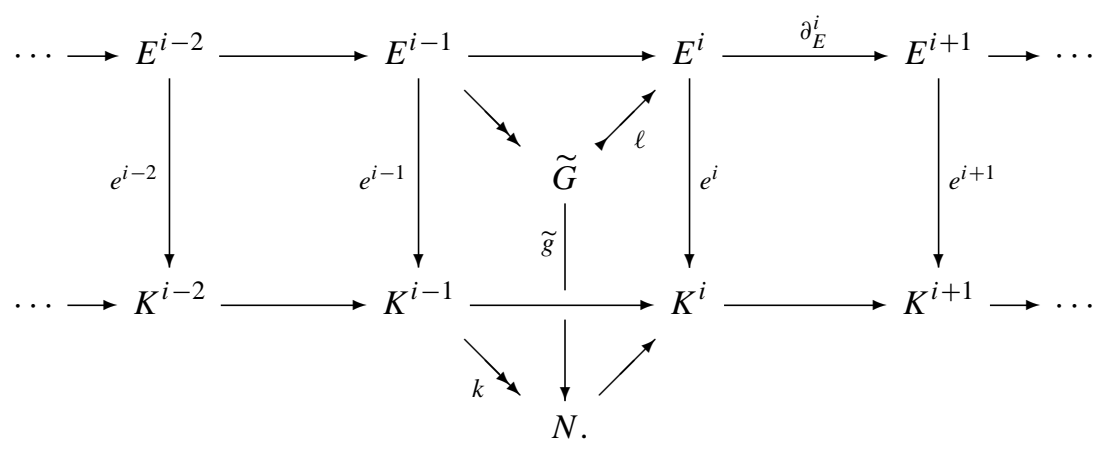

Since Lemma 2.6 says $\operatorname{Hom}_{\mathrm{K}(\operatorname{Pro} A)}(E, K)=0$ for $E$ in $\mathrm{E}(A)$, the chain map $e$ must be null homotopic. Let $\epsilon$ be a null homotopy with $e=\epsilon \partial^{E}+\partial^{K} \epsilon$, consisting of components $E^{j} \stackrel{\epsilon^{j}}{\rightarrow} K^{j-1}$. Then it is straightforward to prove

$$
k \circ\left(\epsilon^{i} \ell\right)=\tilde{g},
$$

so $\widetilde{G} \stackrel{\widetilde{g}}{\rightarrow} N$ has been lifted through $K^{i-1} \stackrel{k}{\rightarrow} N$ as desired.

Remark 2.9. For the next theorem, recall that an augmented Gorenstein projective resolution of an $A$-left-module $M$ is an exact sequence

$$
\cdots \rightarrow G_{2} \rightarrow G_{1} \rightarrow G_{0} \rightarrow M \rightarrow 0,
$$

where the $G_{i}$ are Gorenstein projective modules, which stays exact when one applies the functor $\operatorname{Hom}_{A}(\widetilde{G},-)$ for any Gorenstein projective module $\widetilde{G}$. The complex

$$
G=\cdots \rightarrow G_{2} \rightarrow G_{1} \rightarrow G_{0} \rightarrow 0 \rightarrow \cdots
$$

is then called a Gorenstein projective resolution of $M$. 
Remark 2.10. Recall Construction 2.5 The complex $F$ is in $\mathrm{E}(A)$. In particular it is exact, and therefore the cohomology long exact sequence shows

$$
\mathrm{H}^{i} K=\left\{\begin{array}{cc}
M & \text { for } i=1, \\
0 & \text { for } i \neq 1 .
\end{array}\right.
$$

Hence there is an exact sequence

$$
\cdots \rightarrow K^{-2} \rightarrow K^{-1} \rightarrow K^{0} \rightarrow \operatorname{Ker} \partial_{K}^{1} \rightarrow M \rightarrow 0 .
$$

Theorem 2.11. Let $M$ be an A-left-module. Then the exact sequence from Remark 2.10 is an augmented Gorenstein projective resolution of $M$.

Proof. The modules $K^{0}, K^{-1}, \ldots$ are projective and hence Gorenstein projective.

As for Ker $\partial_{K}^{1}$, observe that in the short exact sequence from Construction 2.5 , the complex $P$ is concentrated in non-positive cohomological degrees, so the modules $P^{1}$ and $P^{2}$ are zero. So in degrees 1 and 2, the short exact sequence gives

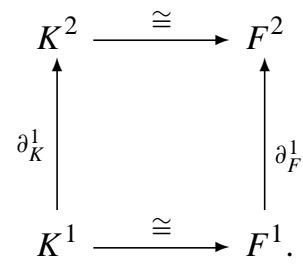

Hence $\operatorname{Ker} \partial_{K}^{1} \cong \operatorname{Ker} \partial_{F}^{1}$, and $\operatorname{Ker} \partial_{F}^{1}$ is Gorenstein projective because $F$ is in $\mathrm{E}(A)$.

To complete the proof, I must show that the exact sequence from Remark 2.10 .

$$
\cdots \rightarrow K^{-2} \rightarrow K^{-1} \rightarrow K^{0} \rightarrow \operatorname{Ker} \partial_{K}^{1} \rightarrow M \rightarrow 0,
$$

remains exact when one applies the functor $\operatorname{Hom}_{A}(\widetilde{G},-)$ for any Gorenstein projective module $\widetilde{G}$.

First, let $i \leq 0$ be an integer and let $\widetilde{G} \stackrel{\widetilde{g}}{\rightarrow} K^{i}$ be a homomorphism whose composition with the subsequent homomorphism in the exact sequence is zero. I must show that $\tilde{g}$ lifts through $K^{i-1} \rightarrow K^{i}$.

I can view $\widetilde{g}$ as a homomorphism $\widetilde{G} \stackrel{\widetilde{g}}{\rightarrow} \operatorname{Ker} \partial_{K}^{i}$, and must then show that $\widetilde{g}$ lifts through the canonical homomorphism $K^{i-1} \rightarrow \operatorname{Ker} \partial_{K}^{i}$. But this follows from Lemma 2.8 applied to

$$
\cdots \rightarrow K^{i-2} \rightarrow K^{i-1} \rightarrow \operatorname{Ker} \partial_{K}^{i} \rightarrow 0 .
$$

Secondly, let $\widetilde{G} \stackrel{\widetilde{g}}{\rightarrow}$ Ker $\partial_{K}^{1}$ be a homomorphism whose composition with the subsequent homomorphism in the exact sequence, $\operatorname{Ker} \partial_{K}^{1} \rightarrow M$, is zero. I must show that $\widetilde{g}$ lifts through $K^{0} \rightarrow \operatorname{Ker} \partial_{K}^{1}$.

I can view $\widetilde{g}$ as a homomorphism $\widetilde{G} \stackrel{\widetilde{g}}{\rightarrow} \operatorname{Im} \partial_{K}^{0}$, and must then show that $\widetilde{g}$ lifts through the canonical homomorphism $K^{0} \rightarrow \operatorname{Im} \partial_{K}^{0}$. But this follows from Lemma 2.8 applied to

$$
\cdots \rightarrow K^{-1} \rightarrow K^{0} \rightarrow \operatorname{Im} \partial_{K}^{0} \rightarrow 0 .
$$


Thirdly, let $\widetilde{G} \stackrel{\widetilde{g}}{\rightarrow} M$ be a homomorphism. I must show that $\widetilde{g}$ lifts through Ker $\partial_{K}^{1} \rightarrow$ $M$. However, from the data given I can construct a commutative diagram

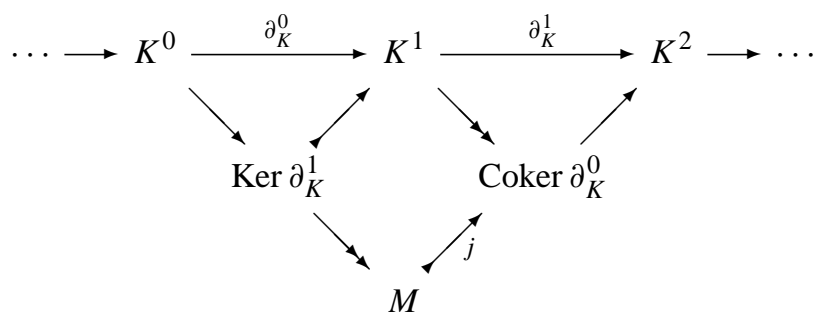

and by applying Lemma 2.8 to

$$
\cdots \rightarrow K^{0} \rightarrow K^{1} \rightarrow \text { Coker } \partial_{K}^{0} \rightarrow 0
$$

I find that $\widetilde{G} \stackrel{j \widetilde{g}}{\rightarrow}$ Coker $\partial_{K}^{0}$ lifts through $K^{1} \rightarrow$ Coker $\partial_{K}^{0}$. It is a small diagram exercise to see that hence $\widetilde{G} \stackrel{\widetilde{g}}{\rightarrow} M$ lifts through Ker $\partial_{K}^{1} \rightarrow M$ as desired.

Let me close the section with the following easy consequence.

Remark 2.12. Recall that a Gorenstein projective precover of an A-left-module $M$ is a homomorphism $G \stackrel{g}{\rightarrow} M$ where $G$ is a Gorenstein projective module, so that if $\widetilde{G}$ is any Gorenstein projective module with a homomorphism $\widetilde{G} \stackrel{\widetilde{g}}{\rightarrow} M$, then $\widetilde{g}$ lifts through $g$,

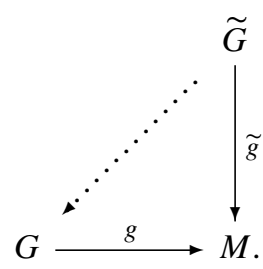

Corollary 2.13. Each A-left-module has a Gorenstein projective precover.

Proof. It follows from Theorem 2.11 that the homomorphism Ker $\partial_{K}^{1} \rightarrow M$ is a Gorenstein projective precover.

\section{Tate cohomology}

This section defines Tate cohomology groups when $e^{\text {! }}$ exists and goes on to show some basic properties: A short exact sequence in either variable gives a long exact sequence of Tate cohomology groups, the Tate cohomology groups coincide with the previously defined Tate cohomology groups from [3] and [26] when these exist, and classical Tate cohomology is the special case $\widehat{\operatorname{Ext}}_{k G}^{i}(k, N)$ of the new definition. Finally, it is proved that the Tate cohomology groups fit into the long exact sequence (3) from the introduction.

Recall that Setup 2.1 remains in force. 
Remark 3.1. It is classical that the category of $A$-left-modules $\operatorname{Mod}(A)$ is equivalent to the full subcategory of K(Pro $A$ ) consisting of projective resolutions of $A$-left-modules. Let

$$
\text { res }: \operatorname{Mod}(A) \rightarrow \mathrm{K}(\operatorname{Pro} A)
$$

be a functor implementing the equivalence.

Definition 3.2. If $M$ and $N$ are A-left-modules, then the Tate cohomology groups of $M$ and $N$ are

$$
\widehat{\operatorname{Ext}}^{i}(M, N)=\mathrm{H}^{i} \operatorname{Hom}_{A}\left(e^{!} \operatorname{res} M, N\right) \text {. }
$$

Remark 3.3. As pointed out in Remark 2.3 the complex $e^{!}$res $M$ can be thought of as the best approximation to $M$ by a complete projective resolution. So taking Hom into $N$ and taking cohomology is the obvious way to get Tate cohomology groups.

Proposition 3.4. Let

$$
0 \rightarrow M^{\prime} \rightarrow M \rightarrow M^{\prime \prime} \rightarrow 0 \text { and } 0 \rightarrow N^{\prime} \rightarrow N \rightarrow N^{\prime \prime} \rightarrow 0
$$

be short exact sequences of A-left-modules. Then there are natural long exact sequences

$$
\cdots \rightarrow \widehat{\operatorname{Ext}}^{i}\left(M^{\prime \prime}, N\right) \rightarrow \widehat{\operatorname{Ext}}^{i}(M, N) \rightarrow \widehat{\operatorname{Ext}}^{i}\left(M^{\prime}, N\right) \rightarrow \cdots
$$

and

$$
\cdots \rightarrow \widehat{\operatorname{Ext}}^{i}\left(M, N^{\prime}\right) \rightarrow \widehat{\operatorname{Ext}}^{i}(M, N) \rightarrow \widehat{\operatorname{Ext}}^{i}\left(M, N^{\prime \prime}\right) \rightarrow \cdots
$$

Proof. It is well known that the first short exact sequence in the proposition results in a distinguished triangle in $\mathrm{K}$ (Pro $A)$,

$$
\operatorname{res} M^{\prime} \rightarrow \operatorname{res} M \rightarrow \operatorname{res} M^{\prime \prime} \rightarrow \text {. }
$$

Since $e_{*}$ is a triangulated functor, so is its adjoint $e^{!}$by [23, Lem. 5.3.6], so there is also a distinguished triangle in $\mathrm{E}(A)$,

$$
e^{!} \operatorname{res} M^{\prime} \rightarrow e^{!} \operatorname{res} M \rightarrow e^{!} \operatorname{res} M^{\prime \prime} \rightarrow
$$

This again results in a distinguished triangle

$$
\operatorname{Hom}_{A}\left(e^{!} \operatorname{res} M^{\prime \prime}, N\right) \rightarrow \operatorname{Hom}_{A}\left(e^{!} \operatorname{res} M, N\right) \rightarrow \operatorname{Hom}_{A}\left(e^{!} \operatorname{res} M^{\prime}, N\right) \rightarrow
$$

whose cohomology long exact sequence is the first long exact sequence in the proposition.

The complex $e^{!}$res $M$ is in $\mathrm{E}(A)$ so consists of projective modules, so the second short exact sequence in the proposition gives a short exact sequence of complexes

$$
0 \rightarrow \operatorname{Hom}_{A}\left(e^{!} \operatorname{res} M, N^{\prime}\right) \rightarrow \operatorname{Hom}_{A}\left(e^{!} \operatorname{res} M, N\right) \rightarrow \operatorname{Hom}_{A}\left(e^{!} \operatorname{res} M, N^{\prime \prime}\right) \rightarrow 0
$$

whose cohomology long exact sequence is the second long exact sequence in the proposition. 
Remark 3.5. If $M$ and $N$ are $A$-left-modules, then there is a previous definition from [3] and [26] by which the Tate cohomology groups of $M$ and $N$ are

$$
\mathrm{H}^{i} \operatorname{Hom}_{A}(T, N)
$$

where $T$ is a complete projective resolution of $M$. This means that $T$ is in $\mathrm{E}(A)$ and sits in a diagram of chain maps

$$
T \stackrel{t}{\rightarrow} P \rightarrow M
$$

where $P \rightarrow M$ is a projective resolution and where $T^{i} \stackrel{t^{i}}{\rightarrow} P^{i}$ is bijective for $i \ll 0$.

Note that not all $A$-left-modules have complete projective resolutions. In fact, the ones that do are exactly the ones which have finite Gorenstein projective dimension by [26, Thm. 3.4].

Lemma 3.6. Let $M$ be an A-left-module which has a projective resolution $P$ and a complete projective resolution $T$. Then

$$
e^{!} P \cong T
$$

in $\mathrm{K}(\operatorname{Pro} A)$.

Proof. All projective resolutions of $M$ are isomorphic in K(Pro $A$ ), so I may as well prove the lemma for the specific projective resolution $P$ from equation (6).

By applying Construction 2.4 to the chain map $T \stackrel{t}{\rightarrow} P$ in cohomological degrees larger than some number, I can assume that $t$ is surjective. Hence there is a short exact sequence of complexes

$$
0 \rightarrow K \rightarrow T \stackrel{t}{\rightarrow} P \rightarrow 0
$$

Since both $T$ and $P$ consist of projective modules, the sequence is semi-split and $K$ also consists of projective modules. Moreover, by assumption, $T^{i} \stackrel{t^{i}}{\rightarrow} P^{i}$ is bijective for $i \ll 0$, so $K^{i}=0$ for $i \ll 0$. So $K$ is a left-bounded complex of projective modules.

Now let $E$ be in $\mathrm{E}(A)$. In particular, $\operatorname{Hom}_{A}(E, Q)$ is exact when $Q$ is a projective module. It is classical that $\operatorname{Hom}_{A}(E, K)$ is then also exact, because $K$ is a left-bounded complex of projective modules. Indeed, this follows by an argument analogous to the one which shows that if $X$ is an exact complex and $I$ is a left-bounded complex of injective modules, then $\operatorname{Hom}_{A}(X, I)$ is exact.

Since the sequence (7) is semi-split, it stays exact under the functor $\operatorname{Hom}_{A}(E,-)$. So there is a short exact sequence of complexes

$$
0 \rightarrow \operatorname{Hom}_{A}(E, K) \rightarrow \operatorname{Hom}_{A}(E, T) \rightarrow \operatorname{Hom}_{A}(E, P) \rightarrow 0 .
$$

Since $\operatorname{Hom}_{A}(E, K)$ is exact, the cohomology long exact sequence shows that there is an isomorphism

$$
\mathrm{H}^{0} \operatorname{Hom}_{A}(E, T) \cong \mathrm{H}^{0} \operatorname{Hom}_{A}(E, P)
$$

which is natural in $E$. That is, there is a natural isomorphism

$$
\operatorname{Hom}_{\mathrm{K}(\operatorname{Pro} A)}(E, T) \cong \operatorname{Hom}_{\mathrm{K}(\operatorname{Pro} A)}(E, P)
$$


which can also be written

$$
\operatorname{Hom}_{E(A)}(E, T) \cong \operatorname{Hom}_{K}(\operatorname{Pro} A)(E, P)
$$

because $E$ and $T$ are in $\mathrm{E}(A)$.

On the other hand, I also have a natural isomorphism

$$
\operatorname{Hom}_{\mathrm{K}(\operatorname{Pro} A)}(E, P)=\operatorname{Hom}_{\mathrm{K}(\operatorname{Pro} A)}\left(e_{*} E, P\right) \cong \operatorname{Hom}_{\mathrm{E}(A)}\left(E, e^{!} P\right) .
$$

Combining the last two equations gives a natural isomorphism

$$
\operatorname{Hom}_{E(A)}(E, T) \cong \operatorname{Hom}_{E(A)}\left(E, e^{!} P\right),
$$

proving $T \cong e^{!} P$ as desired.

Proposition 3.7. Let $M$ be an A-left-module which has a complete projective resolution T. Then the Tate cohomology groups (see Definition 3.2) coincide with the previously defined Tate cohomology groups from [3] and [26] (see Remark 3.5).

Proof. Lemma 3.6 shows that the projective resolution res $M$ of $M$ satisfies $e^{!}$res $M \cong T$. Combining this with the formulae in Definition 3.2 and Remark 3.5 proves the proposition.

Proposition 3.8. Let $k$ be a field, $G$ a finite group, and $N$ a finite-dimensional $k$-linear representation of $G$. Then the Tate cohomology groups

$$
\widehat{\operatorname{Ext}}_{k G}^{i}(k, N)
$$

are defined, and they are isomorphic to the classical Tate cohomology groups of $N$,

$$
\widehat{\mathrm{H}}^{i}(G ; N) \text {. }
$$

Proof. The group algebra $k G$ is a finite-dimensional $k$-algebra. It is clearly left-coherent and right-noetherian, and since it is in fact self-injective, it is clear that ${ }_{k G} k G_{k G}$ is a dualizing complex (cf. Setup 1.4).

Hence $e^{!}$exists over $k G$ by Theorem 1.10 and so the Tate cohomology groups $\widehat{\operatorname{Ext}}_{k G}^{i}(k, N)$ are defined.

The previously defined Tate cohomology groups from [3] and [26] also exist, and they are isomorphic to the classical Tate cohomology groups $\widehat{\mathrm{H}}^{i}(G ; N)$ by [3, Exam. 5.1] with $k$ in place of $\mathbb{Z}$.

But the Tate cohomology groups $\widehat{\operatorname{Ext}}_{k G}^{i}(k, N)$ coincide with the Tate cohomology groups from [3] and [26] by Proposition 3.7, so the present proposition follows.

Definition 3.9. If $M$ and $N$ are A-left-modules, then the Gorenstein Ext groups of $M$ and $N$ are

$$
\operatorname{Ext}_{\mathscr{G}}^{i}(M, N)=\mathrm{H}^{i} \operatorname{Hom}_{A}(G, N)
$$

where $G$ is a Gorenstein projective resolution of $M$ (cf. Remark 2.9). 
Remark 3.10. The resolution $G$ exists by Theorem 2.11. Note that $\operatorname{Ext}_{\mathscr{G}}^{i}(-,-)$ is a well defined bifunctor; see [3] or [11] for this and other properties.

Construction 3.11. Consider the short exact sequence from Construction 2.5.

$$
0 \rightarrow K \rightarrow F \rightarrow P \rightarrow 0
$$

where $P$ is a projective resolution of the $A$-left-module $M$ and where $F=e_{*} e^{!} P$. Truncating the complexes $K$ and $F$ gives a new short exact sequence of complexes,

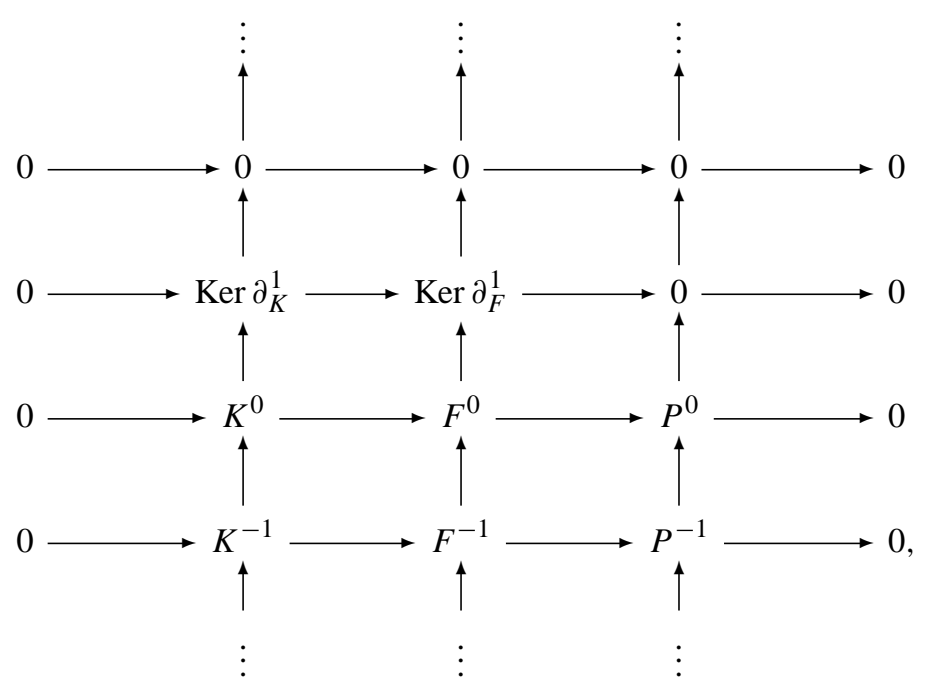

which I will denote

$$
0 \rightarrow K^{\prime} \rightarrow F^{\prime} \rightarrow P \rightarrow 0
$$

Theorem 3.12. Let $M$ and $N$ be A-left-modules. Then there is a long exact sequence

$$
\begin{aligned}
0 \rightarrow \operatorname{Ext}_{\mathscr{G}}^{1}(M, N) \rightarrow & \operatorname{Ext}^{1}(M, N) \rightarrow \widehat{\operatorname{Ext}}^{1}(M, N) \rightarrow \cdots \\
& \rightarrow \operatorname{Ext}_{\mathscr{G}}^{i}(M, N) \rightarrow \operatorname{Ext}^{i}(M, N) \rightarrow \widehat{\operatorname{Ext}}^{i}(M, N) \rightarrow \cdots,
\end{aligned}
$$

natural in $M$ and $N$.

Proof. Consider the short exact sequence (8) from Construction 3.11 . The complex $P$ is a projective resolution of $M$ and in order to make everything natural in $M$, I can clearly suppose

$$
P=\operatorname{res} M
$$

where res $M$ from Remark 3.1 is a projective resolution depending functorially on $M$. Since $P=$ res $M$ consists of projective modules, the short exact sequence $(8)$ is semisplit and therefore stays exact under the functor $\operatorname{Hom}_{A}(-, N)$. So there is a short exact sequence of complexes

$$
0 \rightarrow \operatorname{Hom}_{A}(\operatorname{res} M, N) \rightarrow \operatorname{Hom}_{A}\left(F^{\prime}, N\right) \rightarrow \operatorname{Hom}_{A}\left(K^{\prime}, N\right) \rightarrow 0 .
$$


Since res $M$ is a projective resolution of $M$, I have

$$
\mathrm{H}^{i} \operatorname{Hom}_{A}(\operatorname{res} M, N)=\operatorname{Ext}^{i}(M, N)
$$

for each $i$.

The complex

$$
F=e_{*} e^{!} P=e^{!} \operatorname{res} M
$$

is in $\mathrm{E}(A)$, so it is exact, so

$$
F^{\prime}=\cdots \rightarrow F^{-1} \rightarrow F^{0} \rightarrow \operatorname{Ker} \partial_{F}^{1} \rightarrow 0 \rightarrow \cdots
$$

is also exact, and hence $\mathrm{H}^{0} \operatorname{Hom}_{A}\left(F^{\prime}, N\right)=0$. On the other hand, the form of $F^{\prime}$ makes it clear that

$$
\mathrm{H}^{i} \operatorname{Hom}_{A}\left(F^{\prime}, N\right)=\mathrm{H}^{i} \operatorname{Hom}_{A}(F, N)=\mathrm{H}^{i} \operatorname{Hom}_{A}\left(e^{!} \operatorname{res} M, N\right)=\widehat{\operatorname{Ext}}^{i}(M, N)
$$

for $i \geq 1$.

Finally, Theorem 2.11 says that

$$
K^{\prime}=\cdots \rightarrow K^{-1} \rightarrow K^{0} \rightarrow \operatorname{Ker} \partial_{K}^{1} \rightarrow 0 \rightarrow \cdots
$$

is a Gorenstein projective resolution of $M$, shifted one step to the right. Hence

$$
\mathrm{H}^{i} \operatorname{Hom}_{A}\left(K^{\prime}, N\right)=\operatorname{Ext}_{\mathscr{G}}^{i+1}(M, N)
$$

for $i \geq-1$.

So looking at the cohomology long exact sequence of (9), starting with $\mathrm{H}^{0} \operatorname{Hom}_{A}\left(F^{\prime}, N\right)=0$, gives

$$
0 \rightarrow \operatorname{Ext}_{\mathscr{G}}^{1}(M, N) \rightarrow \operatorname{Ext}^{1}(M, N) \rightarrow \widehat{\operatorname{Ext}}^{1}(M, N) \rightarrow \cdots
$$

as desired.

Acknowledgements. I would like to thank Edgar Enochs and Amnon Neeman for a number of illuminating comments and answers.

The diagrams were typeset with Paul Taylor's diagrams.tex.

\section{References}

[1] Auslander, M., Bridger, M.: Stable module theory. Mem. Amer. Math. Soc. 94 (1969) Zbl 0204.36402 MR 0269685

[2] Avramov, L. L., Foxby, H.-B.: Ring homomorphisms and finite Gorenstein dimension. Proc. London Math. Soc. (3) 75, 241-270 (1997) Zbl 0901.13011 MR 1455856

[3] Avramov, L. L., Martsinkovsky, A.: Absolute, relative, and Tate cohomology of modules of finite Gorenstein dimension. Proc. London Math. Soc. (3) 85, 393-440 (2002) Zbl 1047.16002 MR 1912056

[4] Bousfield, A. K.: The localization of spectra with respect to homology. Topology 18, 257-281 (1979) Zbl 0417.55007 MR 0551009 
[5] Christensen, L. W.: Gorenstein Dimensions. Lecture Notes in Math. 1747, Springer, Berlin (2000) Zbl 0965.13010 MR 1799866

[6] Eilenberg, S., Moore, J. C.: Foundations of relative homological algebra. Mem. Amer. Math. Soc. 55 (1965) Zbl 0129.01101 MR 0178036

[7] Enochs, E. E., Jenda, O. M. G., López-Ramos, J. A.: The existence of Gorenstein flat covers. Math. Scand. 94, 46-62 (2004) Zbl 1061.16003 MR 2032335

[8] Enochs, E. E., López-Ramos, J. A.: Kaplansky classes. Rend. Sem. Mat. Univ. Padova 107, 67-79 (2002) Zbl pre02216807 MR 1926201

[9] Goichot, F.: Homologie de Tate-Vogel équivariante. J. Pure Appl. Algebra 82, 39-64 (1992) Zbl 0767.55004 MR 1181092

[10] Hartshorne, R.: Residues and Duality. Lecture Notes in Math. 20, Springer, Berlin (1966). Notes of a seminar on the work of A. Grothendieck, given at Harvard 1963/64. With an appendix by P. Deligne Zbl 0212.26101 MR 0222093

[11] Holm, H.: Gorenstein derived functors. Proc. Amer. Math. Soc. 132, 1913-1923 (2004) Zbl 1049.16007 MR 2053961

[12] Iyengar, S., Krause, H.: Acyclicity versus total acyclicity for complexes over noetherian rings. Preprint (2005) math.AC/0506265

[13] Jørgensen, P.: Finite flat and projective dimension. Comm. Algebra 33, 2275-2279 (2005) Zbl pre02198700 MR 2153221

[14] Jørgensen, P.: Spectra of modules. J. Algebra 244, 744-784 (2001) Zbl 0998.18007 MR 1859047

[15] Jørgensen, P.: The homotopy category of complexes of projective modules. Adv. Math. 193, 223-232 (2005) Zbl 1068.18012 MR 2132765

[16] Kawasaki, T.: On Macaulayfication of noetherian schemes. Trans. Amer. Math. Soc. 352, 2517-2552 (2000) Zbl 0954.14032 MR 1707481

[17] Krause, H.: Smashing subcategories and the telescope conjecture-an algebraic approach. Invent. Math. 139, 99-133 (2000) Zbl 0937.18013 MR 1728877

[18] Krause, H.: The stable derived category of a noetherian scheme. Compos. Math. 141, 11281162 (2005) Zbl 1090.18006 MR 2157133

[19] Lazard, D.: Autour de la platitude. Bull. Soc. Math. France 97, 81-128 (1969) Zbl 0174.33301 MR 0254100

[20] Martsinkovsky, A.: A remarkable property of the (co)syzygy modules of the residue field of a nonregular local ring. J. Pure Appl. Algebra 110, 9-13 (1996) Zbl 0858.13010 MR 1390669

[21] Martsinkovsky, A.: New homological invariants for modules over local rings, I. J. Pure Appl. Algebra 110, 1-8 (1996) Zbl 0855.13008 MR 1390668

[22] Matsumura, H.: Commutative Ring Theory. Cambridge Stud. Adv. Math. 8, Cambridge Univ. Press, Cambridge (1986) Zbl 0603.13001 MR 0879273

[23] Neeman, A.: Triangulated Categories. Ann. of Math. Stud. 148, Princeton Univ. Press, Princeton (2001) Zbl 0974.18008 MR 1812507

[24] Raynaud, M., Gruson, L.: Critères de platitude et de projectivité. Techniques de "platification" d'un module. Invent. Math. 13, 1-89 (1971) Zbl 0227.14010 MR 0308104

[25] Takahashi, R.: On the category of modules of Gorenstein dimension zero. Math. Z. 251, 249256 (2005) Zbl pre02221890 MR 2191025

[26] Veliche, O.: Gorenstein projective dimension for complexes. Trans. Amer. Math. Soc. 358, 1257-1283 (2006) Zbl 1094.16007 MR 2187653

[27] Wu, Q.-S., J. Zhang, J.: Dualizing complexes over noncommutative local rings. J. Algebra 239, 513-548 (2001) Zbl 1001.16004 MR 1832904

[28] Yekutieli, A., Zhang, J. J.: Rings with Auslander dualizing complexes. J. Algebra 213, 1-51 (1999) Zbl 0948.16006 MR 1674648 